\title{
Hamiltonian analysis of Linearized Extension of Hořava-Lifshitz gravity
}

\author{
Seoktae Koh* and Sunyoung Shin ${ }^{\dagger}$ \\ Center for Quantum Spacetime, Sogang University, Seoul 121-742, Republic of Korea
}

\begin{abstract}
We investigate the Hamiltonian structure of linearized extended Hořava- Lifshitz gravity in a flat cosmological background following the Faddeev-Jackiw's Hamiltonian reduction formalism. The Hamiltonian structure of extended Hořava-Lifshitz gravity is similar to that of the projectable version of original Hořava-Lifshitz gravity, in which there is one primary constraint and so there are two physical degrees of freedom. We also find that extra scalar graviton mode in an inflationary background can be decoupled from the matter field in the infrared (IR) limit, but it is coupled to the matter field in a general cosmological background. But it is necessary to go beyond linear order in order to draw any conclusion of the strong coupling problem.
\end{abstract}

\footnotetext{
* E-mail address: steinkoh@sogang.ac.kr

$\dagger$ E-mail address: shinsy@sogang.ac.kr
} 
The reconciliation of gravity and quantum theory, which is important to understand the very early stage of our Universe and the black hole, is a very challenging task in theoretical physics. Among the several proposals of quantum theory of gravity, recently Hořava[1] proposed a UV complete, non-relativistic gravity theory which is power-counting renormalizable giving up the Lorentz invariance. Since then, many paid attention to this scenario to apply to the black hole [2], cosmology [3] [4] and observational tests[5]. In spite of its many appealing properties, it seems to suffer from many problems [6] [7] such as instability, strong coupling, renormalizability etc.

In order to alleviate the original Hořava-Lifshitz gravity's problem, in [8] the extended version of Hořava-Lifshitz gravity is proposed, in which the new degree of freedom by taking the spatial gradient of a lapse function is introduced without violating the symmetry of the action. They argued that the strong coupling problem of the scalar graviton mode in the IR limit would be solved. There still remains some debate on strong coupling problem in extended version as well as on the physical degrees of freedom [9] [10].

In this paper, following the previous work[11] of one of the authors, we investigate the Hamiltonian structure of linearized extended Hořava-Lifshitz gravity in a cosmological background using the Faddeev-Jackiw approach [12]. First, we derive the quadratic action in the extended Hořava-Lifshitz gravity model and then obtain constraints and Hamiltonian. Through the investigation of the Poisson algebra, the physical degrees of freedom are exactly counted and we analyze the Hamiltonian structures. Next, we obtain the equations of motion of the physical degrees of freedom and finally we briefly comment about the strong coupling issues in our case in the IR limit.

We consider the Arnowitt-Deser-Misner (ADM) metric which is given by

$$
d s^{2}=\left(-N^{2}+N_{i} N^{i}\right) d t^{2}+2 N_{i} d t d x^{i}+\gamma_{i j} d x^{i} d x^{j},
$$

where $N$ is the lapse function, $N_{i}$ are shift vectors, and $\gamma_{i j}$ is the spatial 3 metric. The Hořava-Lifshitz gravity action in the ADM metric with a single scalar field is

$$
\mathcal{S}=\int d^{4} x N \sqrt{\gamma}\left[\frac{1}{2 \kappa^{2}}\left(K_{i j} K^{i j}-\lambda K^{2}\right)-\mathcal{V}+\frac{1}{2 N^{2}}\left(\dot{\phi}-N^{i} \partial_{i} \phi\right)^{2}-Z(\phi)-V(\phi)\right] .
$$

The extrinsic curvature $K_{i j}$ and its trace are written in terms of the ADM metric (11) as

$$
\begin{aligned}
K_{i j} & =\frac{1}{2 N}\left(\partial_{i} N_{j}+\partial_{j} N_{i}-\frac{\partial \gamma_{i j}}{\partial t}\right), \\
K & =\gamma^{i j} K_{i j}=K_{i}^{i} .
\end{aligned}
$$


For extended Hořava-Lifshitz gravity [8], the gravitational potential term $\mathcal{V}$ in the action (2) depends on $\gamma_{i j}$, its spatial derivative and on 3-dimensional vector $a_{i}$ constructed from the lapse function $N(t, \mathbf{x})$ as

$$
a_{i}=\frac{\partial_{i} N(t, \mathbf{x})}{N(t, \mathbf{x})}
$$

which represents the proper acceleration of the vector field of unit normals to the foliation surfaces [6]. Under the anisotropic scaling transformations

$$
\mathbf{x} \rightarrow l \mathbf{x}, \quad t \rightarrow l^{z} t
$$

the $z=3$ theory in the UV is power-counting renormalizable, so the potential in the action (2) can have at most 6 -th order spatial derivative terms. With these spirits, the gravitational potential for extended Hořava-Lifshitz gravity can take the form

$$
\begin{aligned}
\mathcal{V}= & -\xi R-\alpha a_{i} a^{i}+f_{1} R^{2}+f_{2} R_{i j} R^{i j}+f_{3} R \partial_{i} a^{i}+f_{4} a_{i} \partial^{2} a^{i} \\
& +g_{1}\left(\partial_{i} R\right)^{2}+g_{2} \partial_{i} R_{j k} \partial^{i} R^{j k}+g_{3} \partial^{2} R \partial_{i} a^{i}+g_{4} a_{i} \partial^{4} a^{i},
\end{aligned}
$$

where $\xi, \alpha, f_{n}, g_{n}$ are constants and $\partial^{2}=\partial_{i} \partial^{i} . R$ and $R_{i j}$ are 3-dimensional Ricci scalar and Ricci tensor, respectively. Since we are interested in the linear analysis of extend HořavaLifshitz gravity, the only terms in (77) relevant to the linear analysis on a flat cosmological background are included [8] [13]. Most general gravitational potential form in extended Hořava-Lifshitz gravity can be found in Ref. [8]. It is known that the action (2) with the gravitational potential (7) is invariant under the foliation conserving transformations

$$
\mathbf{x} \rightarrow \mathbf{x}+g(t, \mathbf{x}), \quad t \rightarrow t+f(t)
$$

The $Z(\phi)$ in (2) is the matter part potential constructed from the spatial derivative of a scalar field, which is given by

$$
Z(\phi)=\sum_{n=1}^{3} \xi_{n} \partial_{i}^{(n)} \phi \partial^{i(n)} \phi .
$$

The superscript $(n)$ denotes the $n$-th spatial derivative.

In order to derive the background and linear perturbation equations of motion by varying the action, we expand the metric and the scalar field to the linear order as

$$
\begin{aligned}
N & =a(\eta)(1+\Phi) \\
N_{i} & =a(\eta)^{2} \partial_{i} \beta \\
\gamma_{i j} & =a(\eta)^{2}\left(\delta_{i j}+h_{i j}\right)=a(\eta)^{2}\left((1-2 \mathcal{R}) \delta_{i j}+2\left(\partial_{i} \partial_{j}-\frac{1}{3} \delta_{i j} \partial^{2}\right) E\right),
\end{aligned}
$$


and

$$
\phi(\eta, x)=\phi_{0}(\eta)+\delta \phi(\eta, x)
$$

where the parameter $a(\eta)$ is a scale factor and $\eta$ is a conformal time. In this paper, we only consider the scalar mode perturbations.

From the linear order of the action (2)

$$
\begin{aligned}
\delta_{1} \mathcal{S}= & \int d^{4} x a^{2}\left[\left\{-\frac{3(1-3 \lambda)}{2 \kappa^{2}} \mathcal{H}^{2}-\frac{1}{2} \phi_{0}^{\prime 2}-a^{2} V_{0}\right\} \Phi+\left\{-\frac{(1-3 \lambda)}{4 \kappa^{2}}\left(\mathcal{H}^{2}+2 \mathcal{H}^{\prime}\right)\right.\right. \\
& \left.\left.+\frac{1}{2}\left(\frac{1}{2} \phi_{0}^{\prime 2}-a^{2} V_{0}\right)\right\} h_{k}^{k}+\left\{-\phi_{0}^{\prime \prime}-2 \mathcal{H} \phi_{0}^{\prime}-a^{2} V_{\phi}\right\} \delta \phi\right],
\end{aligned}
$$

where $\mathcal{H}=\frac{a^{\prime}}{a}$ and the prime denotes the derivative with respect to $\eta$, we can obtain the background equation of motion in a flat cosmological background

$$
\begin{aligned}
& \frac{3(1-3 \lambda)}{2 \kappa^{2}} \mathcal{H}^{2}=-\left(\frac{1}{2} \phi_{0}^{\prime 2}+a^{2} V_{0}\right), \\
& \frac{(1-3 \lambda)}{2 \kappa^{2}}\left(\mathcal{H}^{2}+2 \mathcal{H}^{\prime}\right)=\frac{1}{2} \phi_{0}^{\prime 2}-a^{2} V_{0}, \\
& \phi_{0}^{\prime \prime}+2 \mathcal{H} \phi_{0}^{\prime}+a^{2} V_{\phi}=0 .
\end{aligned}
$$

By expanding the action up to 2nd order in terms of the perturbed quantities, the quadratic action yields

$$
\begin{aligned}
\delta_{2} \mathcal{S}= & \int d^{4} x a^{2}\left[\frac{1}{2 \kappa^{2}}(1-3 \lambda)\left\{3 \mathcal{H}^{2} \Phi^{2}+2 \mathcal{H} \Phi \partial^{2}\left(\beta-E^{\prime}\right)+6 \mathcal{H} \Phi \psi^{\prime}+3 \psi^{2}+2 \psi^{\prime} \partial^{2}\left(\beta-E^{\prime}\right)\right\}\right. \\
& +\frac{1}{2 \kappa^{2}}(1-\lambda)\left[\partial^{2}\left(\beta-E^{\prime}\right)\right]^{2}-\alpha \Phi \partial^{2} \Phi-\frac{1}{a^{2}}\left(\partial^{2} \psi\right)^{2}\left(16 f_{1}+6 f_{2}\right)-f_{3} \frac{4}{a^{2}} \partial^{2} \psi \partial^{2} \Phi \\
& +\frac{f_{4}}{a^{2}} \partial^{2} \Phi \partial^{2} \Phi+\frac{1}{a^{4}}\left(16 g_{1}+6 g_{2}\right) \partial^{2} \psi\left(\partial^{2}\right)^{2} \psi-g_{3} \frac{4}{a^{4}}\left(\partial^{2}\right)^{2} \psi \partial^{2} \Phi+g_{4} \frac{1}{a^{4}} \partial^{2} \Phi \partial^{2} \partial^{2} \Phi \\
& +2 \xi(2 \Phi-\psi) \partial^{2} \psi+\frac{1}{2} \delta \phi^{\prime 2}-\phi_{0}^{\prime} \Phi \delta \phi^{\prime}+\frac{1}{2} \phi_{0}^{\prime 2} \Phi^{2}-a^{2} \delta Z-\frac{1}{2} a^{2} V_{\phi \phi}(\delta \phi)^{2}-a^{2} V_{\phi} \Phi \delta \phi \\
& \left.+3 \phi_{0}^{\prime} \psi^{\prime} \delta \phi+\phi_{0}^{\prime} \delta \phi \partial^{2}\left(\beta-E^{\prime}\right)\right],
\end{aligned}
$$

where $\psi$ is defined as

$$
\psi=\mathcal{R}+\frac{1}{3} \partial^{2} E
$$

The quadratic action (16) is invariant under the foliation conserving transformation (8)), so the action can be treated as a constraint system. In a constraint system, it is important to classify the constraints properly and to count exact physical degrees of freedom. For this 
purpose, Faddeev-Jackiw approach [12], in which the phase space can be reduced by solving the constraints and finally the quadratic action can be expressed in terms only of the true physical degrees of freedom, seems to be powerful for linear analysis and in this work we use it to analyze the constraint system described by the quadratic action (16).

First, the conjugate momenta for the canonical variables $\psi, \delta \phi$ and $E$ are calculated by definition as

$$
\begin{aligned}
\Pi^{\phi} & =\frac{\delta\left(\delta_{2} S\right)}{\delta\left(\delta \phi^{\prime}\right)}=a^{2}\left(\delta \phi^{\prime}-\phi_{0}^{\prime} \Phi\right), \\
\Pi^{\psi} & =\frac{\delta\left(\delta_{2} S\right)}{\delta \psi^{\prime}}=a^{2} \frac{1-3 \lambda}{\kappa^{2}}\left[3 \mathcal{H} \Phi+\partial^{2}\left(\beta-E^{\prime}\right)+3 \psi^{\prime}\right]+3 a^{2} \phi_{0}^{\prime} \delta \phi, \\
\Pi^{E} & =\frac{\delta\left(\delta_{2} S\right)}{\delta\left(\partial^{2} E^{\prime}\right)}=-a^{2} \frac{1-3 \lambda}{\kappa^{2}}\left(\mathcal{H} \Phi+\psi^{\prime}\right)-a^{2} \frac{1-\lambda}{\kappa^{2}} \partial^{2}\left(\beta-E^{\prime}\right)-a^{2} \phi_{0}^{\prime} \delta \phi .
\end{aligned}
$$

Using these conjugate momenta, the quadratic action (16) under the Legendre transformation becomes

$$
\delta_{2} \mathcal{S}=\int d^{4} x\left[\Pi^{\psi} \psi^{\prime}+\Pi^{\phi} \delta \phi^{\prime}+\Pi^{E} \partial^{2} E^{\prime}-\mathcal{H}_{c}-\mathcal{C}_{0} \Phi-\Phi \Sigma \Phi-\mathcal{C}_{1} \partial^{2} \beta\right]
$$

where

$$
\begin{aligned}
\mathcal{C}_{0}= & -\mathcal{H} \Pi^{\psi}+\phi_{0}^{\prime} \Pi^{\phi}-4\left\{\xi a^{2} \partial^{2}-f_{3}\left(\partial^{2}\right)^{2}-g_{3} \frac{1}{a^{2}}\left(\partial^{2}\right)^{3}\right\} \psi+\left(a^{4} V_{\phi}+3 a^{2} \mathcal{H} \phi_{0}^{\prime}\right) \delta \phi \\
\mathcal{C}_{1}= & \Pi^{E} \\
\mathcal{H}_{c}= & \frac{(1-\lambda) \kappa^{2}}{4(1-3 \lambda) a^{2}}\left(\Pi^{\psi}\right)^{2}+\frac{3 \kappa^{2}}{4 a^{2}}\left(\Pi^{E}\right)^{2}+\frac{1}{2 a^{2}}\left(\Pi^{\phi}\right)^{2}+\frac{\kappa^{2}}{2 a^{2}} \Pi^{\psi} \Pi^{E}-\frac{\kappa^{2}}{(1-3 \lambda)} \phi_{0}^{\prime} \delta \phi \Pi^{\psi} \\
& +2 \xi a^{2} \psi \partial^{2} \psi+\left(16 f_{1}+6 f_{2}\right)\left(\partial^{2} \psi\right)^{2}-\frac{1}{a^{2}}\left(16 g_{1}+6 g_{2}\right) \partial^{2} \psi\left(\partial^{2}\right)^{2} \psi \\
& +a^{4} \delta Z+\frac{1}{2} a^{4} V_{\phi \phi}(\delta \phi)^{2}+\frac{3 \kappa^{2}}{2(1-3 \lambda)} a^{2} \phi_{0}^{\prime 2} \delta \phi^{2} \\
\Sigma= & a^{2} \alpha \partial^{2}-f_{4}\left(\partial^{2}\right)^{2}-\frac{g_{4}}{a^{2}}\left(\partial^{2}\right)^{3} .
\end{aligned}
$$

As seen from (21), $\partial^{2} \beta$ appear linearly without any time derivative, so its coefficient $\mathcal{C}_{1}$ turns out the primary constraint which is expected to vanish. $\mathcal{C}_{0}$ is, however, not a constraint [11], even if $\Phi$ does not have any time derivative terms in the action, because of the $\Phi$ squared term, $\Phi \Sigma \Phi$. Hence it should be determined from the equation of motion for the auxiliary field $\Phi$,

$$
\begin{aligned}
\frac{\delta\left(\delta_{2} \mathcal{S}\right)}{\delta \Phi\left(t^{\prime}, y\right)} & =\int d^{4} x\left[-\mathcal{C}_{0} \delta(x-y) \delta\left(t-t^{\prime}\right)-2 \Sigma \Phi \delta(x-y) \delta\left(t-t^{\prime}\right)\right] \\
& =-\mathcal{C}_{0}-2 \Sigma \Phi=0
\end{aligned}
$$


Therefore we obtain

$$
\Phi=-\frac{\mathcal{C}_{0}}{2 \Sigma}
$$

Following the Faddeev-Jackiw procedure[12], we can reduce the phase space by solving the constraints $\left(\mathcal{C}_{1}=\Pi^{E}=0\right)$, then finally we obtain the quadratic action including only physical degrees of freedom

$$
\delta_{2} \mathcal{S}=\int d^{4} x\left[\Pi^{\psi} \psi^{\prime}+\Pi^{\phi} \delta \phi^{\prime}-\mathcal{H}_{*}+\frac{\mathcal{C}_{0}^{2}}{4 \Sigma}\right]
$$

where

$$
\begin{aligned}
\mathcal{H}_{*}= & \frac{(1-\lambda) \kappa^{2}}{4(1-3 \lambda) a^{2}}\left(\Pi^{\psi}\right)^{2}+\frac{1}{2 a^{2}}\left(\Pi^{\phi}\right)^{2}-\frac{\kappa^{2}}{(1-3 \lambda)} \phi_{0}^{\prime} \delta \phi \Pi^{\psi} \\
& +2 \xi a^{2} \psi \partial^{2} \psi+\left(16 f_{1}+6 f_{2}\right)\left(\partial^{2} \psi\right)^{2}-\frac{1}{a^{2}}\left(16 g_{1}+6 g_{2}\right) \partial^{2} \psi\left(\partial^{2}\right)^{2} \psi \\
& +a^{4} \delta Z+\frac{1}{2} a^{4} V_{\phi \phi}(\delta \phi)^{2}+\frac{3 \kappa^{2}}{2(1-3 \lambda)} a^{2} \phi_{0}^{\prime 2} \delta \phi^{2} .
\end{aligned}
$$

There is no more constraint so we can not reduce the phase space further. As a result, we find that there are two physical degrees of freedom. This result is similar to that of the projectable version of original Hořava-Lifshitz gravity [11], in which the lapse function $N$ is a function of only time and $\mathcal{C}_{0}$ is also not a primary constraint, so there are two physical degrees of freedom.

In order to compare with the conventional Hamiltonian formalism, we count the physical degrees of freedom from the quadratic action (21). From the beginning there are six canonical variables i.e. $\psi, \phi, E$ and their conjugate momenta and one primary first class constraint $\mathcal{C}_{1}$, so we have two physical degrees of freedom $\left(=\frac{1}{2}(6-2 \times 1)\right)$, that is $\psi$ and $\delta \phi$.

Next, we discuss the gauge invariant quantities under the coordinate transformations, $x^{\mu} \rightarrow x^{\mu}+\xi^{\mu}$. In (extended) Hořava-Lifshitz gravity, $\xi^{0}(t)$ is a function of only time, so the foliation conserving diffeomorphism invariant symmetry is required instead of the full diffeomorphism invariant symmetry under the gauge transformations. $\xi^{i}$ can be decomposed as a longitudinal component $\left(\partial^{i} \xi\right)$ and a transverse component $\left(\xi_{T}^{i}\right)$ in which $\xi_{T}^{i}$ satisfies $\partial_{i} \xi_{T}^{i}=0$. In the Hamiltonian constraint system, the first class constraints play the role of the generators of the gauge transformations[14]. In the present work, because we have only one primary constraint, $\mathcal{C}_{1}$ is the only generator of the gauge transformations. Hence given perturbed quantity $f$, we can calculate the gauge transformation as

$$
\delta_{\xi} f=\left\{f, \xi \mathcal{C}_{1}\right\}_{P}
$$


where the subscript $P$ denotes the Poisson brackets. We find that $\psi$ and $\delta \phi$ are the gauge invariant quantities themselves $\left(\delta_{\xi} \psi=\delta_{\xi} \delta \phi=0\right)$ and $E$ is a pure gauge mode $\left(\delta_{\xi} E=\xi\right)$.

The Hamilton's equations are

$$
\begin{aligned}
\delta \phi^{\prime}= & \left\{\delta \phi, \mathcal{H}_{T}\right\}_{P}=\frac{1}{a^{2}} \Pi^{\phi}-\frac{\phi_{0}^{\prime}}{2 \Sigma} \mathcal{C}_{0}, \\
\psi^{\prime}= & \frac{(1-\lambda) \kappa^{2}}{2(1-3 \lambda) a^{2}} \Pi^{\psi}-\frac{\kappa^{2}}{(1-3 \lambda)} \phi_{0}^{\prime} \delta \phi+\frac{\mathcal{H}}{2 \Sigma} \mathcal{C}_{0}, \\
\Pi^{\phi \prime}= & \frac{\kappa^{2}}{(1-3 \lambda)} \phi_{0}^{\prime} \Pi^{\psi}-a^{4} \delta Z_{\delta \phi}-a^{4} V_{\phi \phi} \delta \phi-\frac{3 \kappa^{2}}{(1-3 \lambda)} a^{2} \phi_{0}^{\prime 2} \delta \phi \\
& +\frac{1}{2 \Sigma}\left(a^{4} V_{\phi}+3 a^{2} \mathcal{H} \phi_{0}^{\prime}\right) \mathcal{C}_{0}, \\
\Pi^{\psi \prime}= & -4 \xi a^{2} \partial^{2} \psi-4\left(8 f_{1}+3 f_{2}\right)\left(\partial^{2}\right)^{2} \psi+\frac{4}{a^{2}}\left(8 g_{1}+3 g_{2}\right)\left(\partial^{2}\right)^{3} \psi \\
& +\frac{1}{2 \Sigma}\left(-4 \xi a^{2} \partial^{2}+4 f_{3}\left(\partial^{2}\right)^{2}+\frac{4 g_{3}}{a^{2}}\left(\partial^{2}\right)^{3}\right) \mathcal{C}_{0},
\end{aligned}
$$

where

$$
\mathcal{H}_{T}=\mathcal{H}_{*}-\mathcal{C}_{0}^{2} / 4 \Sigma
$$

The second order differential equations for $\delta \phi$ and $\psi$ are obtained from the Hamilton's equations (31)-(34)

$$
\begin{aligned}
& \delta \phi^{\prime \prime}+2 \mathcal{H} \delta \phi^{\prime}+\frac{\kappa^{2}}{(1-\lambda)} \phi_{0}^{\prime 2} \delta \phi+a^{2} \delta Z_{\delta \phi}+a^{2} V_{\phi \phi} \delta \phi \\
= & \frac{2}{(1-\lambda)} \phi_{0}^{\prime} \psi^{\prime}-\frac{(1-3 \lambda)}{(1-\lambda)} \mathcal{H} \phi_{0}^{\prime} \Phi-2 a^{2} V_{\phi} \Phi+\phi_{0}^{\prime} \Phi^{\prime}, \\
& \psi^{\prime \prime}+2 \mathcal{H} \psi^{\prime}+\frac{2(1-\lambda) \kappa^{2}}{(1-3 \lambda) a^{2}}\left\{\xi a^{2} \partial^{2}+\left(8 f_{1}+3 f_{2}\right)\left(\partial^{2}\right)^{2}-\frac{1}{a^{2}}\left(8 g_{1}+3 g_{2}\right)\left(\partial^{2}\right)^{3}\right\} \psi \\
= & -\frac{\kappa^{2}}{(1-3 \lambda)} \phi_{0}^{\prime} \delta \phi^{\prime}-\frac{\kappa^{2}}{(1-3 \lambda)}\left(\phi_{0}^{\prime \prime}+2 \mathcal{H} \phi_{0}^{\prime}\right) \delta \phi \\
& -\frac{(1-\lambda) \kappa^{2}}{2(1-3 \lambda) a^{2}}\left\{-4 \xi a^{2} \partial^{2}+4 f_{3}\left(\partial^{2}\right)^{2}+\frac{4 g_{3}}{a^{2}}\left(\partial^{2}\right)^{3}\right\} \Phi \\
& -\mathcal{H} \Phi^{\prime}-\left(2 \mathcal{H}^{2}+\mathcal{H}^{\prime}\right) \Phi .
\end{aligned}
$$

Here, from (27) $\Phi$ becomes

$$
\Phi=\frac{\mathcal{D}_{3}}{\mathcal{D}_{1}} \psi^{\prime}-\frac{a^{2} \phi_{0}^{\prime}}{\mathcal{D}_{1}} \delta \phi^{\prime}-\frac{\mathcal{D}_{4}}{\mathcal{D}_{1}} \delta \phi+\frac{\mathcal{A}}{\mathcal{D}_{1}} \psi
$$

with the functions defined by

$$
\mathcal{D}_{1}=2 \Sigma-\mathcal{D}_{2}
$$




$$
\begin{aligned}
\mathcal{D}_{2} & =\frac{2(1-3 \lambda) a^{2}}{(1-\lambda) \kappa^{2}} \mathcal{H}^{2}+a^{2} \phi_{0}^{\prime 2}, \\
\mathcal{D}_{3} & =\frac{2(1-3 \lambda) a^{2}}{(1-\lambda) \kappa^{2}} \mathcal{H}, \\
\mathcal{D}_{4} & =\frac{(1-3 \lambda)}{(1-\lambda)} a^{2} \mathcal{H} \phi_{0}^{\prime}+a^{4} V_{\phi}, \\
\mathcal{A} & =4\left\{\xi a^{2} \partial^{2}-f_{3}\left(\partial^{2}\right)^{2}-\frac{g_{3}}{a^{2}}\left(\partial^{2}\right)^{3}\right\} .
\end{aligned}
$$

Finally, we briefly discuss about the strong coupling of the scalar graviton mode in the IR limit. In the IR limit where the spatial derivative terms can be neglected, $\Sigma$ and $\mathcal{A}$ are negligible, so we get a relation $\mathcal{D}_{1} \simeq-\mathcal{D}_{2}$. The Lagrangian density of the action (28) then becomes

$$
\begin{aligned}
\mathcal{L}= & \left(\frac{(1-3 \lambda) a^{2}}{(1-\lambda) \kappa^{2}}-\frac{1}{2} \frac{\mathcal{D}_{3}^{2}}{\mathcal{D}_{2}}\right) \psi^{\prime 2}+\frac{1}{2} a^{2}\left(1-\frac{a^{2} \phi_{0}^{\prime 2}}{\mathcal{D}_{2}}\right) \delta \phi^{\prime 2}+a^{2} \phi_{0}^{\prime} \frac{\mathcal{D}_{3}}{\mathcal{D}_{2}} \psi^{\prime} \delta \phi^{\prime} \\
& +\left(\frac{\mathcal{D}_{3} \mathcal{D}_{4}}{\mathcal{D}_{2}}+\frac{2 a^{2}}{(1-\lambda)} \phi_{0}^{\prime}\right) \delta \phi \psi^{\prime}+\frac{1}{2}\left(a^{2} \phi_{0}^{\prime} \frac{\mathcal{D}_{4}}{\mathcal{D}_{2}}\right)^{\prime} \delta \phi^{2}-\frac{1}{2} \frac{\mathcal{D}_{4}^{2}}{\mathcal{D}_{2}} \delta \phi^{2} \\
& -2 \xi a^{2} \psi \partial^{2} \psi-\left(16 f_{1}+6 f_{2}\right)\left(\partial^{2} \psi\right)^{2}+\frac{1}{a^{2}}\left(16 g_{1}+6 g_{2}\right) \psi\left(\partial^{2}\right)^{3} \psi \\
& -a^{4} \delta Z-\frac{1}{2} a^{4} V_{\phi \phi}(\delta \phi)^{2}-\frac{\kappa^{2} a^{2}}{2(1-\lambda)} \phi_{0}^{\prime 2} \delta \phi^{2} .
\end{aligned}
$$

In a general flat cosmological background, the scalar graviton mode $\psi$ is found to be coupled to the matter such as $\psi^{\prime} \delta \phi^{\prime}$ and $\delta \phi \psi^{\prime}$. In addition, in the limit of $\lambda \rightarrow 1$ the coefficient of $\psi^{\prime 2}$ is finite, so the scalar graviton mode turns out to be propagating mode.

As a special case, we consider an inflationary accelerating background. By taking the slow-roll approximations $\left(\dot{\phi}_{0}^{2} \ll H^{2} \sim \kappa^{2} V, \ddot{\phi}_{0} \ll 3 H \dot{\phi}_{0}\right.$ where $H=\frac{\dot{a}}{a}$ and dot is a derivative with respect to $t), \mathcal{D}_{2}$ and $\mathcal{D}_{4}$ approximate to

$$
\mathcal{D}_{2} \simeq \frac{2(1-3 \lambda) a^{2}}{(1-\lambda) \kappa^{2}} \mathcal{H}^{2}, \quad \mathcal{D}_{4} \simeq-\frac{2 a^{2}}{(1-\lambda)} \mathcal{H} \phi_{0}^{\prime} .
$$

The Lagrangian density (44) then yields

$$
\begin{aligned}
\mathcal{L}= & \frac{1}{2} a^{2}\left(1-\frac{(1-\lambda) \kappa^{2}}{2(1-3 \lambda)} \frac{\phi_{0}^{\prime 2}}{\mathcal{H}^{2}}\right) \delta \phi^{\prime 2}-\frac{\kappa^{2}}{2(1-3 \lambda)}\left(\frac{a^{2} \phi_{0}^{\prime 2}}{\mathcal{H}}\right)^{\prime} \delta \phi^{2} \\
& -2 \xi a^{2} \psi \partial^{2} \psi-\left(16 f_{1}+6 f_{2}\right)\left(\partial^{2} \psi\right)^{2}+\frac{1}{a^{2}}\left(16 g_{1}+6 g_{2}\right) \psi\left(\partial^{2}\right)^{3} \psi \\
& -a^{4} \delta Z-\frac{1}{2} a^{4} V_{\phi \phi}(\delta \phi)^{2}-\frac{3 \kappa^{2} a^{2}}{2(1-3 \lambda)} \phi_{0}^{\prime 2} \delta \phi^{2} .
\end{aligned}
$$

We ignore the coefficient of the term $\psi^{\prime} \delta \phi^{\prime}$, because

$$
\frac{a \phi_{0}^{\prime}}{\mathcal{H}} \propto \frac{m_{p}^{2} V_{\phi}}{V} \ll 1
$$


Thus, the scalar graviton mode $\psi$ decouples from the matter field completely and is also non-propagating mode because no kinetic term of $\psi$ exists.

Although we discuss about the strong coupling in the IR limit when $\lambda \rightarrow 1$ at the linear order, in fact it seems to be necessary to go beyond linear order to tell whether a mode of interest is strongly coupled or not [4], which is out of the scope of the present paper.

In this paper, we have investigated the Hamiltonian structure of the extended version of Hořava-Lifshitz gravity in a flat cosmological background using Faddeev-Jackiw approach at linear order. The gravitational potential in extension of Hořava-Lifshitz gravity depends on 3-dimensional vector field $a_{i}$ constructed from the lapse function $N(t, \mathbf{x})$ as well as on 3-spatial metric $\gamma_{i j}$ and its derivative.

Since the quadratic action includes $\Phi^{2}$ term, $\mathcal{C}_{0}$, the coefficient of $\Phi$, is not a constraint, even if $\Phi$ does not have any time derivative terms in the action. This implies that $\Phi$ could be determined from the equation of motion. As a result, there is only one primary constraint, $\mathcal{C}_{1}$, so we have two physical degrees of freedom and one of them corresponds to the scalar graviton mode. This Hamiltonian structure is similar to that of the projectable version of

original Hořava-Lifshitz gravity [11] in which the quadratic action includes $\Phi^{2}$ terms and $\mathcal{C}_{0}=0$ in a flat background so there are two physical degrees of freedom.

Further, we have found that in a general flat cosmological background the scalar graviton mode is coupled to the scalar field and is propagating mode in the $\lambda \rightarrow 1$ limit. But as a special case, in an inflationary background with the slow-roll approximation, it can be decoupled completely and corresponds to the non-propagating mode. But the linear order perturbations are not enough to talk about the strong coupling issues, so it would be interesting to go beyond linear order in order to tell whether the mode is strongly coupled or not. And although the linear instability issues (see Ref. [4] for a strong coupling and a linear instability in original Hořava-Lifshitz gravity for a cosmological background) are not treated in this paper, it would also be important questions to be answered in the extended version of Hořava-Lifshitz gravity.

\section{Acknowledgments}

We thank Mu-In Park and Frederico Arroja for useful comments and discussions. We also thank Shinji Mukohyama for useful comments, especially for pointing out the strong 
coupling problem in the IR limit at the nonlinear order. SK thanks the Yukawa Institute for Theoretical Physics at Kyoto University, where this work was completed during the YITPT-10-01 on "Gravity and Cosmology (GC2010)". SK is supported by the National Research Foundation of Korea Grant funded by the Korean Government [NRF-2009-353-C00007]. This work was supported by the National Research Foundation of Korea(NRF) grant funded by the Korea government(MEST) through the Center for Quantum Spacetime(CQUeST) of Sogang University with grant number 2005-0049409 (S. S.).

[1] P. Horava, Phys. Rev. D 79, 084008 (2009) arXiv:0901.3775 [hep-th]].

[2] H. Lu, J. Mei and C. N. Pope, Phys. Rev. Lett. 103, 091301 (2009) [arXiv:0904.1595 [hep-th]]; R. G. Cai, L. M. Cao and N. Ohta, Phys. Rev. D 80, 024003 (2009) arXiv:0904.3670 [hep-th]]; A. Kehagias and K. Sfetsos, Phys. Lett. B 678, 123 (2009) arXiv:0905.0477 [hep-th]].

[3] G. Calcagni, JHEP 0909, 112 (2009) arXiv:0904.0829 [hep-th]];

E. Kiritsis and G. Kofinas, Nucl. Phys. B 821, 467 (2009) [arXiv:0904.1334 [hep-th]];

S. Mukohyama, JCAP 0906, 001 (2009) [arXiv:0904.2190 [hep-th]].

[4] S. Mukohyama, arXiv:1007.5199 [hep-th].

[5] M. i. Park, JCAP 1001, 001 (2010) arXiv:0906.4275 [hep-th]];

S. Dutta and E. N. Saridakis, JCAP 1001, 013 (2010) arXiv:0911.1435 [hep-th]];

S. S. N. Kim, T. Kim and Y. Kim, Phys. Rev. D 80, 124002 (2009) [arXiv:0907.3093 [hep-th]];

S. Mukohyama, K. Nakayama, F. Takahashi and S. Yokoyama, Phys. Lett. B 679, 6 (2009) arXiv:0905.0055 [hep-th]];

T. Takahashi and J. Soda, Phys. Rev. Lett. 102, 231301 (2009) arXiv:0904.0554 [hep-th]];

S. Koh, arXiv:0907.0850 [hep-th].

[6] D. Blas, O. Pujolas and S. Sibiryakov, JHEP 0910, 029 (2009) arXiv:0906.3046 [hep-th]].

[7] M. Li and Y. Pang, JHEP 0908, 015 (2009) [arXiv:0905.2751 [hep-th]];

K. Koyama and F. Arroja, arXiv:0910.1998 [hep-th];

M. Henneaux, A. Kleinschmidt and G. L. Gomez, arXiv:0912.0399 [hep-th].

A. Papazoglou and T. P. Sotiriou, Phys. Lett. B 685, 197 (2010) arXiv:0911.1299 [hep-th]];

D. Blas, O. Pujolas and S. Sibiryakov, arXiv:0912.0550 [hep-th].

[8] D. Blas, O. Pujolas and S. Sibiryakov, Phys. Rev. Lett. 104, 181302 (2010) arXiv:0909.3525 
[hep-th]].

[9] J. Kluson, JHEP 1007, 038 (2010) [arXiv:1004.3428 [hep-th]];

I. Kimpton and A. Padilla, JHEP 1007, 014 (2010) [arXiv:1003.5666 [hep-th]];

D. Blas, O. Pujolas and S. Sibiryakov, Phys. Lett. B 688, 350 (2010) arXiv:0912.0550 [hepth]];

A. Papazoglou and T. P. Sotiriou, Phys. Lett. B 685, 197 (2010) arXiv:0911.1299 [hep-th]].

[10] D. Blas, O. Pujolas and S. Sibiryakov, arXiv:1007.3503 [hep-th].

[11] J. O. Gong, S. Koh and M. Sasaki, arXiv:1002.1429 [hep-th].

[12] L. D. Faddeev and R. Jackiw, Phys. Rev. Lett. 60, 1692 (1988).

[13] T. Kobayashi, Y. Urakawa and M. Yamaguchi, JCAP 1004, 025 (2010) arXiv:1002.3101 [hep-th]].

[14] M. Henneaux and C. Teitelboim, "Quantization of gauge systems," Princeton, USA: Univ. Pr. (1992) $520 p$ 